\title{
Primary dumbbell-shaped Ewing's sarcoma of the cervical vertebra in adults: Four case reports and literature review
}

\author{
QING ZHU, JISHENG ZHANG and JIANRU XIAO
}

Department of Orthopedics, Shanghai Changzheng Hospital, Second Military Medical University, Shanghai 86200, P.R. China

Received October 21, 2011; Accepted December 23, 2011

DOI: 10.3892/ol.2012.550

\begin{abstract}
Ewing's sarcoma is the second most common malignant bone tumor in children and adolescents. The 4 cases described in this study were diagnosed with dumbbell-shaped intraspinal and extraspinal Ewing's sarcomas. The incidence of dumbbell-shaped tumors of this type in the spine is $17.5 \%$. These tumors are often misdiagnosed as neurogenic tumors (schwannoma, neurofibromatosis) or giant cell tumors based on imaging. Radiculopathy is more common than spinal cord compression in Ewing's sarcoma. Preoperative biopsy is strongly recommended. As soon as Ewing's sarcoma is diagnosed by pathology, the treatment should begin with 2-3 cycles of neoadjuvant chemotherapy. Anterior-posterior and posterolateral approaches are both recommended for exposing this tumor. Following surgery, chemotherapy is critical to lessen the rate of recurrence and metastasis and to prolong the survival time. However, radiotherapy should be used with caution, as the spinal cord is sensitive to radiation; local irradiation is suggested. The tumor is difficult to remove en bloc in the cervical spine. It has a high rate of recurrence and metastasis. Therefore, the prognosis of Ewing's sarcoma in the cervical region is poorer compared to that in the thoracic and lumbosacral regions.
\end{abstract}

\section{Introduction}

Ewing's sarcoma/primitive neuroectodermal tumor (PNET) is quite rare. Between 1973 and 2004, the incidence of the disease in the US was 2-93 per 1,000,000 individuals (1). Ewing's sarcoma is the second most common malignant bone tumor in children and adolescents. It affects the pelvic bone and femur, but rarely the cervical spine. The incidence of primary vertebral Ewing's sarcoma is $3.5 \%$ (2). It is thought to arise from neural crest cells (3). In 1984, Jaffe et al described

Correspondence to: Professor Jianru Xiao, Department of Orthopedics, Shanghai Changzheng Hospital, Second Military Medical University, 415 Fengyang Road, Shanghai 86200, P.R. China

E-mail: jianruxiao83@163.com

Key words: Ewing's sarcoma, surgery, cervical vertebra a small round-cell tumor of the bone, designated neuroectodermal tumor of bone (Ewing's sarcoma of bone) (4). This tumor is difficult to diagnose only by hematoxylin and eosin (H\&E) staining.

In this report, all of the 4 cases were diagnosed with dumbbell-shaped intraspinal and extraspinal Ewing's sarcomas.

The study was approved by the research department of Shanghai Changzheng Hospital, China, and the patients involved provided their informed consent.

\section{Case report}

Descriptions of the 4 cases are provided in Table I. The 3 newonset patients received almost the same treatment (8-10 cycles of chemotherapy and local irradiation) following surgery.

Case 1. The patient was a 46-year-old male who experienced neck and shoulder pain for 12 months prior to the discovery of a mass in the neck. Physical examination revealed a mass with tenderness. There was limitation of motion in the cervical vertebra. The force of the left deltoid was approximately level 3 and the force of the other muscles was normal. Diagnostic imaging, including computed tomography (CT) and magnetic resonance imaging (MRI), revealed a dumbbellshaped soft tissue mass beside the C3-C6 vertebra. MRI showed a collapse in the transverses of the $\mathrm{C} 3$ and $\mathrm{C} 4$ vertebra (Fig. 1). A neurogenic tumor was suggested by imaging. After we finished preparation, the patient underwent surgery in June 2008. The procedure involved a piecemeal resection. Anterior-posterior surgeries were performed in this case. At first, the posterior approach was used. The tumor inside the spinal canal was removed. The removed tumor was sent to be frozen in sections. Pathological investigation revealed a small round-cell malignant tumor (Fig. 2). Cisplatin was subsequently used for intraoperative chemotherapy. Following the posterior approach, surgery from the anterior approach was performed (Fig. 3). The extraspinal part of the tumor was removed completely. The nerve root and vertebral artery were protected during the surgery. After surgery, the pathological diagnosis confirmed Ewing's sarcoma. The patient felt that the pain had been significantly relieved and the force of deltoid had been slightly recovered. Although local radiotherapy and chemotherapy were used following surgery, lung metastasis was observed. The patient succumbed to the disease 12 months after surgery. 
Table I. Summary of the 4 cases.

\begin{tabular}{|c|c|c|c|c|}
\hline & Case 1 & Case 2 & Case 3 & Case 4 \\
\hline Gender & Male & Male & Male & Male \\
\hline Age (years) & 46 & 27 & 27 & 24 \\
\hline Clinical manifestations & - & - & - & $\begin{array}{l}\text { Before the first } \\
\text { operation }\end{array}$ \\
\hline Pain & $\begin{array}{l}\text { Mild pain for } \\
12 \text { months }\end{array}$ & $\begin{array}{l}\text { Mild pain for } \\
2 \text { months }\end{array}$ & $\begin{array}{l}\text { Mild pain for } \\
6 \text { months }\end{array}$ & $\begin{array}{l}\text { Mild pain for } \\
1 \text { month }\end{array}$ \\
\hline Neurological impairment & Radiculopathy & Radiculopathy & Radiculopathy & $\begin{array}{c}\text { Spinal cord compression } \\
\text { and radiculopathy }\end{array}$ \\
\hline \multicolumn{5}{|l|}{ Strength } \\
\hline Upper limb & Level 4-5 & Level 3 & Level 2-4 & Level 3-4 \\
\hline Lower limb & Level 5 & Level 5 & Level 5 & Level 3-4 \\
\hline Date of surgery & 6.16 .2008 & 8.12 .2010 & 9.9.2009 & 2.10 .2011 \\
\hline Surgical approach & Anterior-posterior & Anterior-posterior & Anterior-posterior & Posterolateral \\
\hline Blood loss & $1000 \mathrm{ml}$ & $1300 \mathrm{ml}$ & $2000 \mathrm{ml}$ & $800 \mathrm{ml}$ \\
\hline Prognosis & Died in 12 months & Still alive & Still alive & Died in 7 months \\
\hline Metastasis & Lung metastasis & None & None & Brain metastasis \\
\hline
\end{tabular}

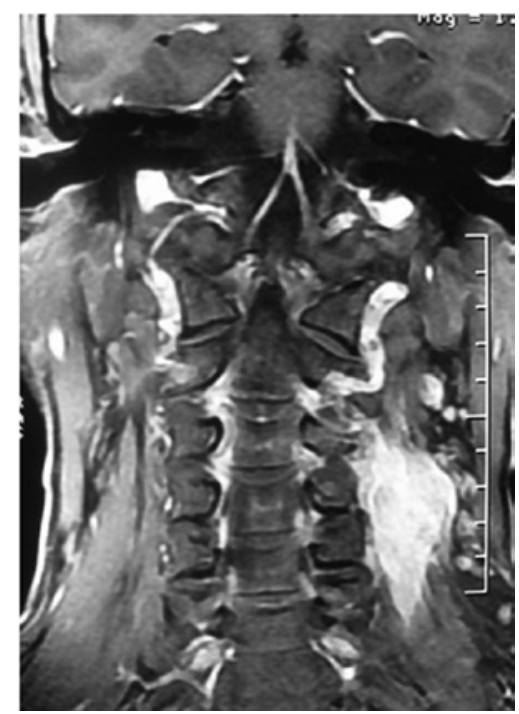

Figure 1. Case 1. Coronal T1-weighted enhanced MRI of cervical spine reveals a dumbbell-shaped intraspinal and extraspinal mass. MRI, magnetic resonance imaging.

Case 2. The patient was a 27-year-old male with neck and shoulder pain for 1 month prior to the discovery of a mass in the neck. Physical examination revealed a mass with tenderness. There was limitation of motion in the cervical vertebra. The force of the muscles of the left upper limb was approximately level 3 and the force of the other muscles was normal. The lower limb showed slight spasticity. Diagnostic imaging included CT and MRI. Imaging revealed a dumbbell-shaped soft tissue mass on the left of the C1-C4 vertebra. MRI showed collapse in the transverses of the C3 vertebra (Fig. 4). A neurogenic tumor was suggested by imaging. After we finished the preparation, the patient underwent surgery in August 2010. The surgery was a piecemeal resection. Anterior-posterior surgery

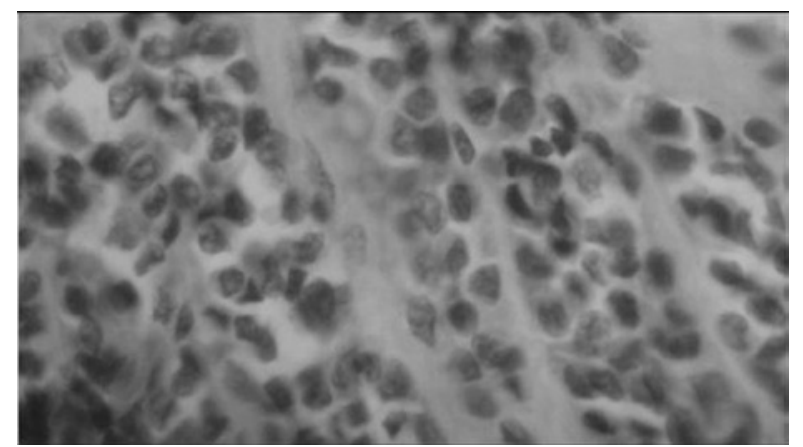

Figure 2.Case 1.Paraffinsection by H\&Estaining.H\&E, hematoxylinandeosin.

was performed in this case. At first, the posterior approach was used. The tumor inside the spinal canal was removed. The removed tumor was sent to be frozen in sections. Pathological investigation revealed a malignant neurogenic tumor. Cisplatin was used for the intraoperative chemotherapy treatment. Following the posterior approach, surgery from the anterior approach was performed. The extraspinal part of the tumor was removed completely. After surgery, the pathological diagnosis confirmed Ewing's sarcoma. The patient felt that the pain had been significantly relieved and the force of the muscles of the left upper limb had slightly recovered. Local radiotherapy and chemotherapy were used following surgery. No recurrence and metastasis have occurred thus far.

Case 3. The patient was a 27-year-old male with neck and shoulder pain, and numbness in the left upper limb for 6 months. Physical examination revealed that there was a limitation of motion in the cervical vertebra. The force of the inner hand muscles of the left limb was approximately level 2 and the force of other muscles of the left limb was approximately level 4. Diagnostic imaging included CT, MRI and 


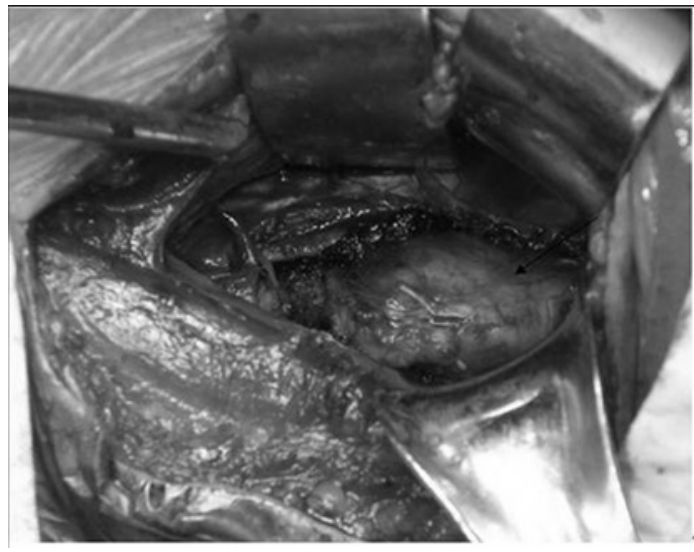

Figure 3. Case 1. The tumor (arrow) is exposed by the anterior approach.

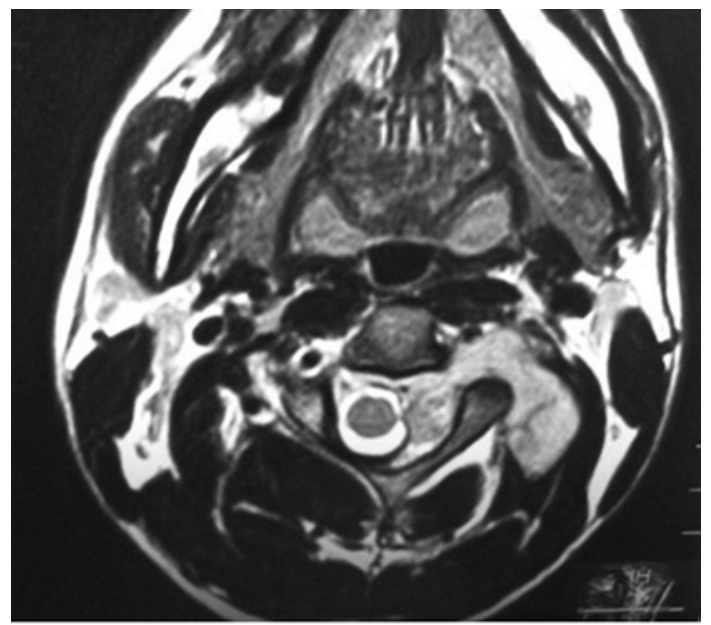

Figure 4. Case 2. Axial T2-weighted MRI of the spine reveals a dumbbell-shaped soft-tissue mass compressing the spinal cord and left nerve root. MRI, magnetic resonance imaging.

PET-CT. The imaging revealed a dumbbell-shaped soft tissue mass on the left of the C7 vertebra. MRI showed collapse in the transverses of $\mathrm{C} 7$ vertebra (Fig. 5). PET-CT showed the FDG was mildly absorbed. A giant cell tumor (GCT) was suggested by PET-CT. After we finished the preparation, the patient underwent surgery in September 2009. The surgery involved piecemeal resection. Anterior-posterior surgery was performed in this case. At first, the anterior approach was used. The extraspinal tumor was removed. The removed tumor was sent to be frozen. Pathological investigation revealed a small round-cell malignant tumor. Cisplatin was used as intraoperative chemotherapy. After the anterior approach, surgery from the posterior approach was performed. The intraspinal part of the tumor was removed completely. The vertebral artery was protected during the surgery; however, the $\mathrm{C} 7$ nerve root was not protected. Following surgery, pathological diagnosis confirmed Ewing's sarcoma (Fig. 6). The patient felt that the pain had been significantly relieved and the force of muscles of the left limb had slightly recovered. Local radiotherapy and chemotherapy were used following surgery. Recurrence and metastasis have not been found to date.

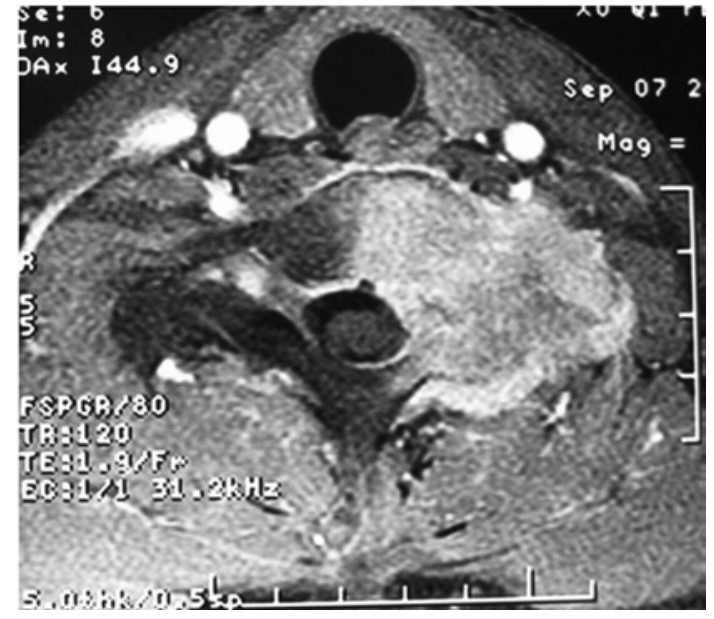

Figure 5. Case 3. Axial T1-weighted enhanced MRI of the C7 vertebra reveals a soft-tissue mass invading the vertebral body and appendix. MRI magnetic resonance imaging.

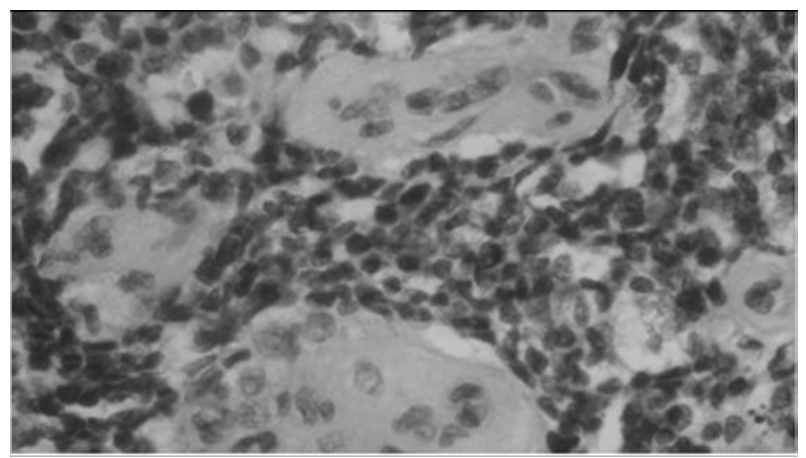

Figure 6. Case 3. paraffin section by $H \& E$ staining. H\&E, hematoxylin and eosin.

Case 4. The patient was a 24-year-old male with neck pain and numbness in the left upper limb for 1 month. The patient had received surgery in December 2009 in another hospital. A total of 7 months later, the patient complained of weakness and numbness in the left limb. The recurrence was found by MRI (Fig. 7). Physical examination revealed that the force of the muscles of the left limb was approximately level 3, and the force of other muscles of the right limb was approximately level 4. Diagnostic imaging included CT and MRI. They revealed a dumbbell-shaped soft tissue mass on the left of the C5 vertebra. MRI showed a collapse in the transverses of the C5 vertebra. After finishing the preparation, the patient underwent surgery in July 2010. The surgery was a piecemeal resection. The posterolateral approach was used. The tumor was located at the ventral side of the spinal cord and was removed carefully. The tightly adherent dura was removed together, then the dura was repaired by soft tissue. Cisplatin was used for intraoperative chemotherapy treatment. After the surgery, the pain had been relieved; the force of muscles, however, had not been recovered. Two weeks later, the patient began to receive chemotherapy. However, recurrence of 


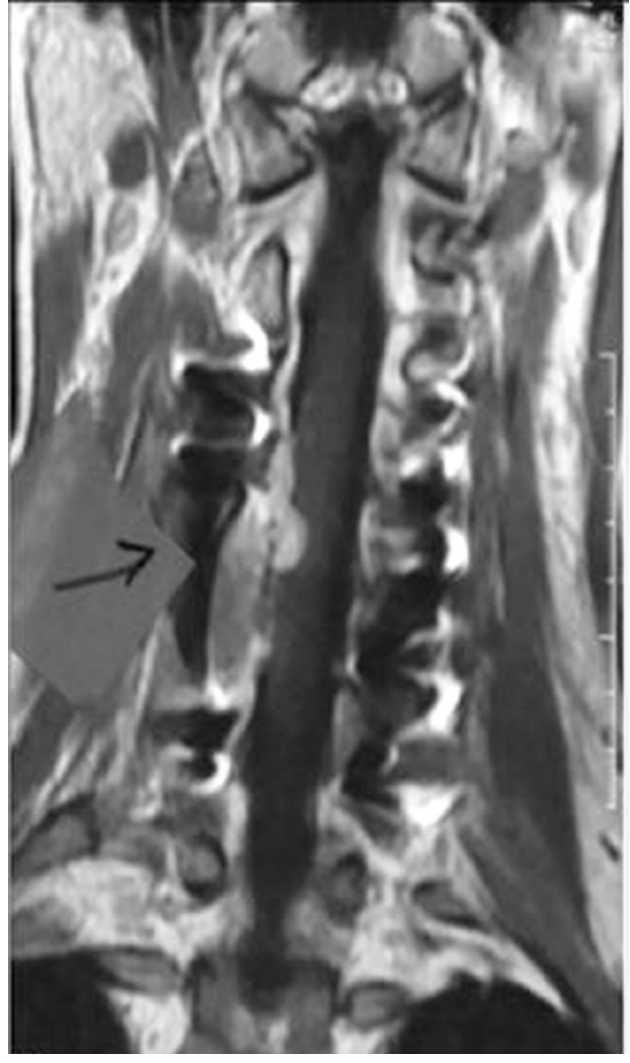

Figure 7. Case 4. Coronal T1-weighted enhanced MRI of the cervical spine reveals the dumbbell-shaped intraspinal and extraspinal mass (arrow). MRI, magnetic resonance imaging.

tumor appeared in the same place 6 months following the initial removal, and the patient succumbed to brain metastasis 7 months after the surgery.

\section{Discussion}

The incidence of dumbbell-shaped tumors in the spine is $17.5 \%$ (118/674). The rate of dumbbell tumors in the cervical spine is significantly higher than that of all spinal cord tumors. Of 118 cases, $69 \%$ of tumors were found to be schwannomas (5).

Clinicopathological studies have revealed that Ewing's sarcoma and PNET have overlapping features, supporting a common histogenesis. Identification of a common translocation $t(11 ; 22)(q 24 ; q 12)(6,7)$ that results in the formation of the EWS-ETS fusion gene (8) in cases of Ewing's sarcoma, PNET and Askin's tumor strongly supports the hypothesis that these tumors are related. Therefore, all of these lesions are now included in the same classification, the Ewing's sarcoma family of tumors (EFTs).

The male to female ratio of Ewing's sarcoma is $1.5: 1.3$. Approximately $80 \%$ of patients are diagnosed with Ewing's sarcoma when they are younger than 20 years old. A total of $50-60 \%$ of the patients are diagnosed during their second decade (9). In the event patients are over 30 years old, the diagnosis of Ewing's sarcoma should be made after exclusion of the possibility of other small round-cell malignant tumors.

Generally, Ewing's sarcoma progresses quite rapidly. The most common clinical manifestation is pain. The 3 new-onset patients in this study suffered mild pain, which was relieved by using non-steroidal anti-inflammatory drugs (NSAIDS). Radiculopathy caused by the tumor was found in all 4 cases. However, spinal cord compression only appeared in the patient with recurrent sarcoma. Due to the rapid progression and the dumbbell-shaped tumor, the nerve root is affected first. Before spinal cord compression appeared, the patients were treated for radiculopathy and the rapidly progressing mass.

Tumor-related osteolysis and periosteal reactions suggest a diagnosis of primary malignant tumor. Typically, Ewing's sarcoma appears as an ill-defined, permeative, or focally moth-eaten, destructive intramedullary lesion accompanied by a periosteal reaction ('onion skin') that affects the diaphysis of long bones (9). MRI is the most sensitive test available for the evaluation of the soft tissue extent of the tumor. The MRI appearance of Ewing's sarcoma is non-specific, with TI-weighted images showing an intermediate signal and T2-weighted images showing an intermediate-to-high signal within the mass (10). In the 3 new-onset patients, neurogenic tumors (schwannoma, neurofibromatosis) and giant cell tumors (GCT) (Case 3) were suggested by MRI.

Prior to surgery, biopsies had not been conducted for the 3 new-onset patients as: i) Ewing's sarcoma is very uncommon in African and Asian populations; ii) Ewing's sarcoma is rare in the population over 20 years of age; iii) it is quite risky to conduct a biopsy in the cervical spine, as the tumor is surrounded by the vertebral artery, spinal cord and nerve root; iv) neurogenic tumors (schwannoma, neurofibromatosis) or GCT were suggested by MRI. For Ewing's sarcoma, the treatment should begin with 2-3 cycles of neoadjuvant chemotherapy. This may help us to achieve marginal resection. In the cervical spine, the tumor is difficult to remove en bloc. Therefore, we propose that biopsy should be conducted although neurogenic tumors (schwannoma, neurofibromatosis) or GCT are suggested by imaging.

Generally, Ewing's sarcoma is sensitive to chemotherapy and irradiation. The proportion of patients whose primary tumors are treated with radiation alone has steadily declined over the past 30 years. This is due to advances in orthopedic surgery and a growing awareness of the late effects of radiation in children, particularly second malignancies and growth disturbances. Patients whose primary tumors are excised have a higher survival rate, although the prognostic effects of site and size complicate the analyses (11).

Surgical en bloc resection, where feasible, is regarded as the best modality for local control (12). Anterior-posterior surgeries were performed in 3 cases in this study. The posterolateral approach was performed in 1 case, which is also recommended. Although en bloc resection was not performed, we did our best to achieve total resection of the tumor. In addition, cisplatin was used for the treatment of intraoperative chemotherapy in order to lessen the rate of recurrence and metastasis.

Ewing's sarcomas are sensitive to chemotherapy and irradiation. In the Cooperative Ewing's Sarcoma Study (CESS)-81, the 5-year relapse-free survival of patients with non-metastatic Ewing's sarcoma after VACD regimen (vincristine, actinomycin-D, cyclophosphamide and doxorubicin) was $55 \%$ (13). In the CESS-86 study, ifosfamide was substituted for doxorubicin in the treatment of tumors with a volume greater than $100 \mathrm{ml}$. The 10-year event-free survival was 52\% (14). In our cases, 
8-10 cycles of chemotherapy were administered after surgery. The use of chemotherapy has greatly improved survival rates for patients with localized Ewing's sarcoma. Hoiwever, it has much less effect on the survival of patients with metastases at diagnosis. Radiotherapy treatment should be used with caution since the spinal cord is sensitive to radiation. Local irradiation is suggested.

The prognosis of Ewing's sarcoma is quite poor. Non-metastatic disease at presentation has a 5-year disease-free survival rate of $70 \%$, whereas patients with metastatic disease at presentation have a 5-year disease-free survival rate of $25 \%$ (15). In our cases, 2 patients were diagnosed with metastases following surgery. The new-onset patients survived for 7 months and the other patient for 12 months.

Recently, a study showed that insulin-like growth factor 1 receptor (IGF1R)-targeted therapies have resulted in responses in a small number of patients with advanced metastatic Ewing's sarcoma (16).

Ewing's sarcoma is the second most common malignant bone tumor in children and adolescents. It affects the pelvic bone and femur, but rarely the cervical vertebra. All of the 4 cases were diagnosed with dumbbell-shaped intraspinal and extraspinal Ewing's sarcomas. It is likely to be misdiagnosed as a neurogenic tumor (schwannoma, neurofibromatosis) or GCT according to imaging. Radiculopathy is more common than spinal cord compression in Ewing's sarcoma. Preoperative biopsy is strongly recommended. As soon as Ewing's sarcoma is diagnosed by pathology, the treatment should begin with 2-3 cycles of neoadjuvant chemotherapy. The anterior-posterior and posterolateral approach are both recommended for exposing this tumor. Following surgery, chemotherapy is critical to lessen the rate of recurrence and metastasis and prolong the survival time. However, radiotherapy treatment should be used cautiously, as the spinal cord is sensitive to radiation. Local irradiation is suggested. The tumor is difficult to be removed en bloc in the cervical spine. It has a high rate of recurrence and metastasis. Therefore, the prognosis of Ewing's sarcomas in the cervical region is poorer than that in the thoracic and lumbosacral region.

\section{References}

1. Esiashvili N, Goodman M and Marcus R: Changes in incidence and survival of Ewing sarcoma patients over the past 3 decades: surveillance epidemiology and end results data. J Pediat Hematol Oncol 30: 425-430, 2008

2. Whitehouse GH and Griffiths GJ: Roentgenological aspects of spinal involvement by primary and metastatic Ewing's tumor. J Can Assoc Radiol 27: 290-297, 1976.

3. Dehner LP: Peripheral and central primitive neuroectodermal tumors: a nosologic concept seeking a consensus. Arch Pathol Lab Med 110: 997-1005, 1986.

4. Jaffe R, Santamaria M, Yunis EJ, et al: The neuroectodermal tumor of bone. Am J Surg Pathol 8: 885-898, 1984.

5. Ozama H, Kokubum S, Aizawa T, Takeshi $\mathrm{H}$ and Chikashi K: Spinal dumbbell tumors: an analysis of a series of 118 cases. J Neurosurg Spine 7: 587-593, 2007.

6. Aurias A, Rimbaut C, Buffe D, Zucker JM and Mazabraud A: Translocation involving chromosome 22 in Ewing's sarcoma: a cytogenetic study of four fresh tumors. Cancer Genet Cytogenet 12: 21-25, 1984.

7. Whang-Peng J, Triche TJ, Knutsen T, Miser J, Douglass EC and Israel MA: Chromosomal translocation in peripheral neuroepithelioma. N Engl J Med 311: 584-585, 1984.

8. Delattre O, Zucman J, Plougastel B, et al: Gene fusion with an ETS DNA-binding domain caused by chromosome translocation in human tumours. Nature 359: 162-165, 1992.

9. Iwamoto Y: Diagnosis and treatment of Ewing's sarcoma. Jpn J Clin Oncol 37: 79-89, 2007.

10. Flemming DJ, Murphy MD, Nallu S and Nicastri AD: Primary Ewing sarcoma of lumbar spine with massive intraspinal extension. Pediatr Neurol 38: 58-60, 2008.

11. Schuck A, Ahrens S, Paulussen M, et al: Local therapy in localized Ewing's tumors: results of 1058 patients treated in the CESS 81, CESS 86, and EICESS 92 trials. Int J Rad Oncol Biol Phys 55: 168-177, 2003.

12. Paulussen M, Bielack S and Jurgens H: Ewing's sarcoma of the bone: ESMO clinical recommendations for diagnosis, treatment and follow-up. Ann Oncol (Suppl 4): 140-142, 2009.

13. Jurgens H, Exner U, Gadner H, et al: Multidisciplinary treatment of primary Ewing's sarcoma of bone. A 6-year experience of a European Cooperative Trial. Cancer 61: 23-32, 1988.

14. Paulussen M, Ahrens S, Dunst J, et al: Localized Ewing's tumor of bone: final results of the cooperative Ewing's Sarcoma Study CESS 86. J Clin Oncol 19: 1818-1829, 2001.

15. Caudill JS and Arndt CA: Diagnosis and management of bone malignancy in adolescence. Adolesc Med 18: 62-78, 2009.

16. Rodon J, DeSantos V, Ferry RJ Jr and- Kurzrock R: Early drug development of inhibitors of the insulin-like growth factor-I receptor pathway: lessons from the first clinical trials. Mol Cancer Ther 7: 2575-2588, 2008. 